\title{
Opposite effect of spin-orbit coupling on condensation and superfluidity
}

\author{
Kezhao Zhou and Zhidong Zhang \\ Shenyang National Laboratory for Materials Science, Institute of Metal Research, \\ and International Centre for Materials Physics, Chinese Academy of Sciences, \\ 72 Wenhua Road, Shenyang 110016, People's Republic of China
}

(Dated: November 15, 2018)

\begin{abstract}
We investigated effects of a Rashba-type spin-orbit coupling (SOC) on the condensed density and superfluid density tensor of a two-component Fermi gas in the BCS-BEC crossover at zero temperature. In anisotropic three dimensions (3D), we found that SOC has an opposite effect on condensation (enhanced) and superfluidity (suppressed in the SOC direction) and this effect becomes most pronounced for very weak interactions and the SOC strength being larger than a characteristic value. Furthermore, as functions of SOC strength, the condensed density changes monotonously for all interaction parameters while the superfluid density has a minimum when the interaction parameter is below a critical value. We also discussed the isotropic two dimensional (2D) case where analytical expressions for the gap and number equations were obtained and the same phenomena was found as that of the 3D case.
\end{abstract}

PACS numbers: 05.30.Fk, 03.75.Hh, 03.75.Kk

Introduction. Spin-orbit coupling (SOC) is a central topic in condensed matter physics. First, it plays an essential role for the realization of nontrivial topological states which are discussed intensively nowadays [1]. Secondly, as was shown by Gor'kov and Rashba [2], SOC can induce a nontrivial spin-triplet pairing field which leads to significant changes in the properties of superconductors [3]. Quite recently, effective SOC was realized for bosonic ${ }^{87} R b$ ultra-cold atoms by dressing two atomic spin states with a pair of lasers [4]. With the anticipation that this novel technique is also applicable to Fermi atoms, practical proposal of generating SOC in fermionic ${ }^{40} \mathrm{~K}$ atoms with tunable interaction through Feshbach Resonance is given in [5].

Motivated by this new progress, effect of SOC on the pairing and superfluid nature of Fermi systems in the BCS-BEC crossover has become a cutting-edge field recently because of its broad interests in condensed matter physics. The spintriplet pairing fields and anisotropic nature of the superfluidity induced by SOC were investigated in [6] and proposal for detecting this phenomenon was given in [7] through measurement of the momentum distribution and single-particle spectral function. On the other hand, SOC significantly enhances the pairing phenomena as was shown by the exact two-body solutions [8] where a new bound state (rashbons) emerges and many-body mean-field calculations [9-11].

In this Letter, we study the effects of SOC on two fundamental quantities: condensation and superfluidity. Condensation is well described by the concept of off-diagonal-longrange-order [12]. However, Landau's approach of calculation of superfluid density (tensor) is only applicable to systems satisfying Galilean transformation [13]. For systems in the presence of SOC obviously violating Galilean transformation, we gave the general method of calculating the superfluid density tensor. Furthermore, we found that at zero temperature, SOC enhances condensation while suppresses superfluidity in both $3 \mathrm{D}$ and $2 \mathrm{D}$. Up to our knowledge, this is the first demonstration of such opposite behaviors of condensation and superfluidity driven by SOC and renews our previous knowledge that these two phenomena change in the same direction with other influencing factors (such as temperature and disorder).

The model.In the presence of SOC, the system of a two-component Fermi gas can be described by the finite temperature grand-partition function $Z=\int d\left[\bar{\psi}_{\sigma}, \psi_{\sigma}\right] \exp \left(-S\left[\bar{\psi}_{\sigma}, \psi_{\sigma}\right]\right) \quad\left(\hbar=k_{B}=1\right.$ through out) where the action $S\left[\bar{\psi}_{\sigma}, \psi_{\sigma}\right]$ is given by $S\left[\bar{\psi}_{\sigma}, \psi_{\sigma}\right]=\int_{0}^{\beta} d \tau \int d^{d} \mathbf{r} \sum_{\sigma}\left[\bar{\psi}_{\sigma} \partial_{\tau} \psi_{\sigma}+\mathcal{H}_{0}+\mathcal{H}_{I}\right]$ with $\beta=1 / T, \sigma=\uparrow, \downarrow$ denoting spin, $\bar{\psi}_{\sigma}, \psi_{\sigma}$ being the Grassmann fields, and $d(=2,3)$ being the dimension. We focus on a Rashba-type SOC [2] and the single particle Hamiltonian density can be written as $\mathcal{H}(\bar{\psi}, \psi)=\bar{\psi}\left(\hat{\xi}_{\mathbf{p}}+\mathcal{H}_{s o}\right) \psi$ where $\psi=\left[\psi_{\uparrow}, \psi_{\downarrow}\right]^{T}$ is the collective fermionic field, the kinetic operator $\hat{\xi}_{\mathbf{p}}=\hat{\mathbf{p}}^{2} /(2 m)-\mu$ with $\mu$ being the chemical potential, the Rashba term $\mathcal{H}_{s o}=\lambda(\hat{\sigma} \times \hat{\mathbf{p}})_{z}$ with $\hat{\sigma}$ being the Pauli matrices and $\lambda$ being the SOC strength. The singlet-channel attractive interaction can be characterized by a contact interaction parameter $g(<0)$ and correspondingly $\mathcal{H}_{I}=g \bar{\psi}_{\uparrow} \bar{\psi}_{\downarrow} \psi_{\downarrow} \psi_{\uparrow}$.

In order to study the Fermi-pairing problems, we employ the Hubbard-Stratonovich transformation [14] to cancel the four-body interaction term $\mathcal{H}_{I}$ by introducing a pairing field $\Delta(\mathbf{r}, \tau)$. After integrating out the fermionic fields, we obtain the effective pairing action as $S_{\text {eff }}[\bar{\Delta}, \Delta]=$ $-\int_{0}^{\beta} d \tau \int d^{d} \mathbf{r}|\Delta(\mathbf{r}, \tau)|^{2} / g-1 / 2 \operatorname{Tr} \ln \left[\mathcal{G}_{\mathbf{r}, \tau}^{-1}\right]$ where the inverse propagator $\mathcal{G}_{\mathbf{r}, \tau}^{-1}$ is

$$
\mathcal{G}_{\mathbf{r}, \tau}^{-1}=\left[\begin{array}{cccc}
\partial_{\tau}+\hat{\xi}_{\mathbf{p}} & \hat{\gamma}_{\mathbf{p}} & 0 & \Delta \\
\hat{\gamma}_{\mathbf{p}}^{*} & \partial_{\tau}+\hat{\xi}_{\mathbf{p}} & -\Delta & 0 \\
0 & -\bar{\Delta} & \partial_{\tau}-\hat{\xi}_{\mathbf{p}} & \hat{\gamma}_{\mathbf{p}}^{*} \\
\bar{\Delta} & 0 & \hat{\gamma}_{\mathbf{p}} & \partial_{\tau}-\hat{\xi}_{\mathbf{p}}
\end{array}\right],
$$

with $\hat{\gamma}_{\mathbf{p}}=\lambda\left(\hat{p}_{y}+i \hat{p}_{x}\right)$.

Mean-field theory. At the mean-field level $\Delta(\mathbf{r}, \tau)=$ $\Delta_{0}$ which is referred to as the gap parameter, the effective pairing action becomes $S_{\text {eff }}[\bar{\Delta}, \Delta]=-\beta V \Delta_{0}^{2} / g-$ $1 / 2 \sum_{\mathbf{p}, i \omega_{n}} \ln \left[\operatorname{det} \mathcal{G}_{\mathbf{p}, i \omega_{n}}^{-1}\right]$ where $\mathcal{G}_{\mathbf{p}, i \omega_{n}}^{-1}$ is the momentum- 
frequency representation of Eq. (1) with $\Delta(\mathbf{r}, \tau)=\Delta_{0}, V$ is the size of the system and $\omega_{n}=(2 n+1) \pi / \beta$ are the Fermi Matsubara frequencies. From $\operatorname{det} \mathcal{G}_{\mathbf{p}, E}^{-1}=0$, the excitation spectrum can be obtained as $E_{\mathbf{p}, \delta}=\sqrt{\left(\xi_{\mathbf{p}}+\delta\left|\gamma_{\mathbf{p}}\right|\right)^{2}+\Delta_{0}^{2}}$ and $E_{\mathbf{p}, \delta}^{\prime}=-E_{\mathbf{p}, \delta}$ where $\xi_{\mathbf{p}}=\epsilon_{\mathbf{p}}-\mu$ with $\epsilon_{\mathbf{p}}=\mathbf{p}^{2} / 2 m$ and $\delta= \pm 1$ is called helicity. Finally, by using the thermodynamic relation $\Omega=-1 / \beta \ln Z_{0}$, we have the thermodynamic potential $\Omega_{0}=-V \Delta_{0}^{2} / g+1 / 2 \sum_{\mathbf{p}, \delta}\left(\xi_{\mathbf{p}}-E_{\mathbf{p}, \delta}\right)-$ $1 / \beta \sum_{\mathbf{p}, \delta= \pm} \ln \left(1+e^{-\beta E_{\mathbf{p}, \delta}}\right)$ from which the gap and number equations are given by

$$
\begin{gathered}
\frac{1}{g}=-\frac{1}{V} \sum_{\mathbf{p}, \delta= \pm} \frac{\tanh \left(\frac{\beta E_{\mathbf{p}, \delta}}{2}\right)}{4 E_{\mathbf{p}, \delta}}, \\
n=\frac{1}{2 V} \sum_{\mathbf{p}, \delta= \pm}\left[1-\frac{\left(\xi_{\mathbf{p}}+\delta\left|\gamma_{\mathbf{p}}\right|\right) \tanh \left(\frac{\beta E_{\mathbf{p}, \delta}}{2}\right)}{E_{\mathbf{p}, \delta}}\right] .
\end{gathered}
$$

Eq. (2) and Eq. (3) are the generalized BCS gap and number equations in the presence of a Rashba-type SOC which have been investigated in detail to study the ground state and finite temperature properties of this novel system. The key discovery is that the increased density of states by SOC plays a crucial role for the understanding of the pairing enhancing phenomena [11]. With these results in mind, we now move on to the calculation and discussion of condensed density and superfluid density tensor.

Condensed density.For the Fermi pairing problems, the condensed density is generally defined as [12] $n_{c}=1 / V \sum_{\mathbf{p}, s s^{\prime}}\left|\left\langle\bar{\psi}_{\mathbf{p}, s} \psi_{-\mathbf{p}, s^{\prime}}\right\rangle\right|^{2}$. For the system considered in this paper, the singlet-channel attractive interaction supports a singlet-pairing field while SOC can simultaneously induce a triplet component. Within the mean-field theory, spin-singlet and -triplet pairing fields are given by [7]: $\left\langle\bar{\psi}_{\mathbf{p}, \uparrow} \psi_{-\mathbf{p}, \downarrow}\right\rangle=$ $\Delta_{0} \sum_{\delta} \tanh \left(\beta E_{\mathbf{p}, \delta} / 2\right) /\left(4 E_{\mathbf{p}, \delta}\right)$ and $\left\langle\bar{\psi}_{\mathbf{p}, \uparrow} \psi_{-\mathbf{p}, \uparrow}\right\rangle=$ $-\Delta_{0}\left(\gamma_{\mathbf{p}} /\left|\gamma_{\mathbf{p}}\right|\right) \sum_{\delta} \delta \tanh \left(\beta E_{\mathbf{p}, \delta} / 2\right) /\left(4 E_{\mathbf{p}, \delta}\right)$, respectively. The spin-singlet contribution to the condensed fraction was first discussed in [15] where it was shown to behave non-monotonously with a minimum as a function of SOC strength for weak enough interaction parameter. In this Letter, we take both pairing components into consideration and the full condensed density becomes

$$
n_{c}=\frac{\Delta_{0}^{2}}{4} \frac{1}{V} \sum_{\mathbf{p}, \delta} \frac{\tanh ^{2}\left(\frac{\beta E_{\mathbf{p}, \delta}}{2}\right)}{E_{\mathbf{p}, \delta}^{2}} .
$$

At zero temperature, repulsive interactions between Fermi pairs (Bosons) result in depletion of the condensate which is a familiar phenomenon for interacting BEC systems.

Superfluid density. Unlike the condensate density, the superfluidity is a kinetic property of the system. By Landau's theory [13], the normal mass of the system can be obtained through the calculation of the total momentum carried by excitations when the system is enforced in a uniform superfluid flow with velocity $\mathbf{v}_{s}$

$$
\mathbf{P}=\sum_{\mathbf{p}, \sigma} \mathbf{p} f\left(E_{\mathbf{p}, \sigma}-\mathbf{p} \cdot \mathbf{v}_{s}\right)
$$

where $\sigma$ is a conserved quantum number which is spin in the absence of SOC, $f(x)=1 /\left(e^{\beta x} \pm 1\right)$ is the Fermi/Bose distribution function depending on the nature of the excitations, and $E_{\mathbf{p}, \sigma}-\mathbf{p} \cdot \mathbf{v}_{s}$ is the excitation spectrum for moving system obtained from Galilean transformation. At zero temperature, no excitations are created at very small $\mathbf{v}_{s}$ and the superfluid density coincides with the total density.

However the situation is dramatically changed in the presence of SOC where Galilean transformation is violated. In order to calculate the response of the system to a uniform superfluid flow in the presence of SOC, instead of using Eq. (5) which is no longer valid, we calculate the increasing of thermodynamic potential $\delta \Omega=\Omega\left(\mathbf{v}_{s}\right)-$ $\Omega(0)=(1 / 2) V \sum_{\alpha \eta} m n_{s, \alpha \eta} v_{\alpha} v_{\eta}$ from which $n_{s, \alpha \eta}=$ $1 /(m V)\left[\partial^{2} \Omega\left(\mathbf{v}_{s}\right) / \partial v_{s, \alpha} \partial v_{s, \eta}\right]_{\mathbf{v}_{s}=0}$ is defined as the superfluid density tensor [16]. A convenient approach of generating such superfluid flow is applying a "phase twist" to the order parameter [17]: $\Delta(\mathbf{r}, \tau)=\Delta_{0} e^{i \mathbf{q} \cdot \mathbf{r}}$ and correspondingly $\mathbf{v}_{s}=\mathbf{q} / 2 \mathrm{~m}$. Therefore the superfluid density tensor can be defined as $n_{s, \alpha \eta}=4 m\left[\partial^{2} \Omega(\mathbf{q}) / \partial q_{\alpha} \partial q_{\eta}\right]_{\mathbf{q}=0}$.

By substitution of $\Delta(\mathbf{r}, \tau)=\Delta_{0} e^{i \mathbf{q} \cdot \mathbf{r}}$ into Eq. (1), the thermodynamic potential for moving system can be obtained as $\Omega(\mathbf{q})=-V \Delta_{0}^{2} / g+\sum_{\mathbf{k}}\left(\tilde{\xi}_{\mathbf{k}}-\mathbf{k} \cdot \mathbf{q} / 2 m\right)-$ $1 /(2 \beta) \sum_{\mathbf{p}, i=1 \sim 4} \ln \left[1+e^{\beta\left(\tilde{E}_{\mathbf{p}, i}-\mathbf{k} \cdot \mathbf{q} / 2 m\right)}\right]$ where $\tilde{\xi}_{\mathbf{p}}=$ $\xi_{\mathbf{p}}+\mathbf{q}^{2} /(8 m)$ and $\tilde{E}_{\mathbf{p}, i}$ are solutions of

$$
\left(\tilde{E}_{\mathbf{p}, i}^{2}-\omega_{\mathbf{p}}\right)^{2}-4\left|\tilde{E}_{\mathbf{p}, i} \gamma_{\mathbf{q} / \mathbf{2}}-\tilde{\xi}_{\mathbf{p}} \gamma_{\mathbf{p}}\right|^{2}+\Lambda_{\mathbf{p}}^{2}=0,
$$

with $\omega_{\mathbf{p}}=\tilde{\xi}_{\mathbf{p}}^{2}+\Delta_{0}^{2}+\left|\gamma_{\mathbf{p}}\right|^{2}-\left|\gamma_{\mathbf{q} / \mathbf{2}}\right|^{2}$, and $\Lambda_{\mathbf{p}}=\operatorname{Im}\left(\gamma_{\mathbf{p}} \gamma_{\mathbf{q}}^{*}\right)$. In the presence of SOC, when the whole system is moving with a uniform velocity, the original four excitation spectrums $\left(E_{\mathbf{p}, \delta},-E_{\mathbf{p}, \delta}\right)$ are strongly coupled and correspondingly Eq. (5) is not well defined now. Superfluidity in systems that violates the Galilean transformation has also been discussed in the bosonic systems in the presence of SOC where the critical velocity has been discussed with the same method used in the calculation of the excitation spectrum for moving systems [18.

Combined with Eq. (6), calculation of the second-order derivative of $\Omega(\mathbf{q})$ with respect to $q_{i}$ is straightforward although tedious. Finally, the superfluid density tensor is obtained as

$$
n_{s, z z}=\frac{N}{V}-\frac{4 m}{V} \sum_{\mathbf{p}, \delta} Y_{\mathbf{p}, \delta}\left(\frac{p_{z}}{2 m}\right)^{2}
$$




$$
\begin{aligned}
n_{s, \|}= & \frac{N}{V}-\frac{2 m}{V} \sum_{\mathbf{k}, \delta} Y_{\mathbf{p}, \delta}\left(\frac{\left|\gamma_{\mathbf{p}}\right|}{2 m \lambda}+\delta \frac{\lambda}{2}\right)^{2} \\
& -\frac{m \lambda^{2}}{4 V} \sum_{\mathbf{p}, \delta} \tanh \left(\frac{\beta E_{\mathbf{p}, \delta}}{2}\right) \frac{\xi_{\mathbf{p}}^{2}+\delta \xi_{\mathbf{p}}\left|\gamma_{\mathbf{p}}\right|+\Delta_{0}^{2}}{\delta \xi_{\mathbf{p}}\left|\gamma_{\mathbf{p}}\right| E_{\mathbf{p}, \delta}}(8)
\end{aligned}
$$

where $Y_{\mathbf{p}, \delta}=\beta f\left(E_{\mathbf{p}, \delta}\right)\left[1-f\left(E_{\mathbf{p}, \delta}\right)\right]$ with $f(x)$ being the Fermi distribution function, $n_{s, x x}=n_{s, y y}=n_{s, \|}$, and $n_{s, \alpha \neq \eta}=0$. In 3D, the anisotropic nature of the superfluid can be evidently seen from $n_{s, z z} \neq n_{s, \|}$. Since SOC does not affect motion in $z$ direction, spin is a conserved quantum number and $n_{s, z z}$ has the same form as that obtained from Eq. (5). However, superfluid motion in $x, y$ direction is dramatically changed by SOC. Most interestingly, a new term (last line in Eq. (8)) emerges due to SOC and it is not zero at $T=0$ which means suppression of superfluidity in $x, y$ direction. The first line of Eq. (8) can be understood in spirit of Landau's theory where momentum carried by excitations $E_{\mathbf{p}, \delta}$ is now shifted by $\delta \lambda m$ due to SOC. At $T=T_{c}$ where $\Delta_{0}=0$, both $n_{s, z}$ and $n_{s, \|}$ equal to zero which is crucial for the correctness of our results. In 2D, the system is isotropic where the superfluid density is only given by $n_{s, \|}$.

With the anticipation that the mean-field theory is sufficient to capture the qualitatively correct physics in the whole BCSBEC region at zero temperature as demonstrated in [19] in the absence of SOC, we only focus on the zero temperature behaviors of the condensed and superfluid density.

Results and discussion. Because, at zero temperature, behaviors of the condensed and superfluid density $\left(n_{s, \|}\right)$ are the same in 3D and 2D cases with only quantitative differences, we will discuss the 3D case in detail and give a brief discussion on the $2 \mathrm{D}$ results.

As usual we regularize the contact interaction parameter $g$ in Eq. (2) by the experimentally related scattering length $a$ through $1 / g=m /(4 \pi a)-1 / V \sum_{\mathbf{p}} 1 /\left(2 \epsilon_{\mathbf{p}}\right)$. With the gap and chemical potential obtained from the self-consistent solutions of Eq. (2) and Eq. (3), we numerically calculate Eq. (4) and Eq. (8) and the results are shown in Fig.1. As can be seen from Fig. 1 a) and (c), the condensed density is always enhanced by SOC. Nevertheless, seen from Fig. 1(a), we can still define a characteristic value roughly located at $\lambda_{c} \approx 0.5 v_{F}$ where $v_{F}=k_{F} / m$ and $k_{F}$ is defined through $k_{F}^{2}=3 \pi^{2} n$. For $\lambda<\lambda_{c}$, condensed fraction defined as $n_{c} / n$ increases only slightly which can also be seen from Fig. 11(c) that the solid orange and dotted blue lines almost coincide with the dash black line (where $\lambda=0$ ). Only when $\lambda>\lambda_{c}$ can $n_{c} / n$ have a significant increase. For $1 / k_{F} a \rightarrow+\infty$, the effect of SOC becomes very weak and $n_{c} / n \rightarrow 1$. For $\lambda_{c} \gg v_{F}$, we also have $n_{c} / n \rightarrow 1$ which agrees with the statement that SOC can produce a bound state and thus induces a crossover even for very weak interactions [8, 11].

On the contrary, the superfluidity is always suppressed by SOC as can be seen in Fig. 1 b). Furthermore, as a function of $\lambda$, superfluid fraction $n_{s, \|} / n$ varies non-monotonously with a minimum located at $\lambda_{c}^{\prime}$ (denoted by red points in Fig. 1(b)). This minimum exists for interaction parameter below

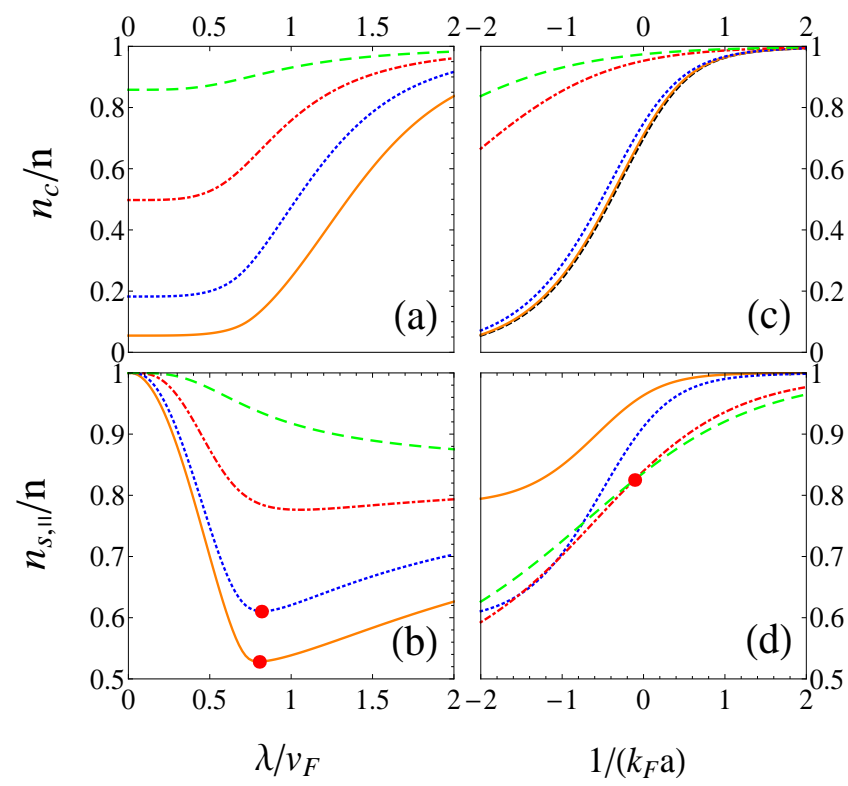

FIG. 1: (Color online) Condensed and superfluid fraction as functions of $\lambda / v_{F}$ and $1 /\left(k_{F} a\right)$ in 3D. In (a) and (b), $1 /\left(k_{F} a\right)$ are given as: $-2,-1.2,-0.4$ and 0.4 for the same line species from below. In (c) and (d), $\lambda / v_{F}$ are set to be 0.4 for solid orange lines, 0.6 for dotted blue lines, 1.6 for dot-dashed red lines and 2.0 for media-dashed green lines. Dashed black line in (c) corresponds to $\lambda=0$. Red points in (b) and (d) are explained in text.

a well-defined critical value $1 / k_{F} a_{c} \approx-0.13$ which can be determined in Fig. 11d) as the rightmost red crossing point. However, we find that SOC never destroys the superfluid completely for all interaction parameters. The minimum superfluid fraction $\left(n_{s, \|} / n\right)_{\min } \rightarrow 0.5$ for $1 / k_{F} a \rightarrow-\infty$. When $1 / k_{F} a>1 / k_{F} a_{c}$, this monotonic behavior disappears and $n_{s, \|} / n$ decreases with increasing $\lambda$.

The opposite behavior of condensation and superfluidity controlled by SOC strength is found to be most pronounced for very weak interaction parameters and $\lambda>\max \left(\lambda_{c}, \lambda_{c}^{\prime}\right)$. As can be seen from the solid orange lines in Fig. 1 a) and (b), for $\lambda=2 v_{F}$, the condensed fraction $n_{c} / n$ is increased by 0.78 and the superfluid density is suppressed by 0.38 . For $\lambda<\max \left(\lambda_{c}, \lambda_{c}^{\prime}\right)$, the superfluid density decreases quickly to its minimum value while the condensed density changes only very slightly.

In $2 \mathrm{D}$, divergence of the integral over momenta can be cured by substituting $1 / g=-1 / V \sum_{\mathbf{p}} 1 /\left(2 \epsilon_{\mathbf{p}}+E_{B}\right)$ into Eq. (2) where $E_{B}$ is the binding energy and becomes the controlling parameter for the BCS-BEC crossover problem. With dimensionless parameter given by $\tilde{\lambda}=\lambda / v_{F}, \tilde{\mu}=\mu / E_{F}, \tilde{\Delta}_{0}=\Delta_{0} / E_{F}$, $\tilde{E}_{B}=E_{B} / E_{F}$ where $E_{F}=k_{F}^{2} / 2 m$ and $k_{F}$ is defined through $k_{F}^{2}=2 \pi n$, Eq. (2) and Eq. (3) have analytical expressions as $1=\left(\tilde{\mu}+\sqrt{\tilde{\mu}^{2}+\tilde{\Delta}_{0}^{2}}\right) / 2+$ $\tilde{\lambda}^{2}\left(1+\tilde{\mu} / \sqrt{\tilde{\mu}^{2}+\tilde{\Delta}_{0}^{2}}\right)+H\left(\tilde{\mu}, \tilde{\Delta}_{0}, \tilde{\lambda}\right), \quad \ln E_{b}$ 


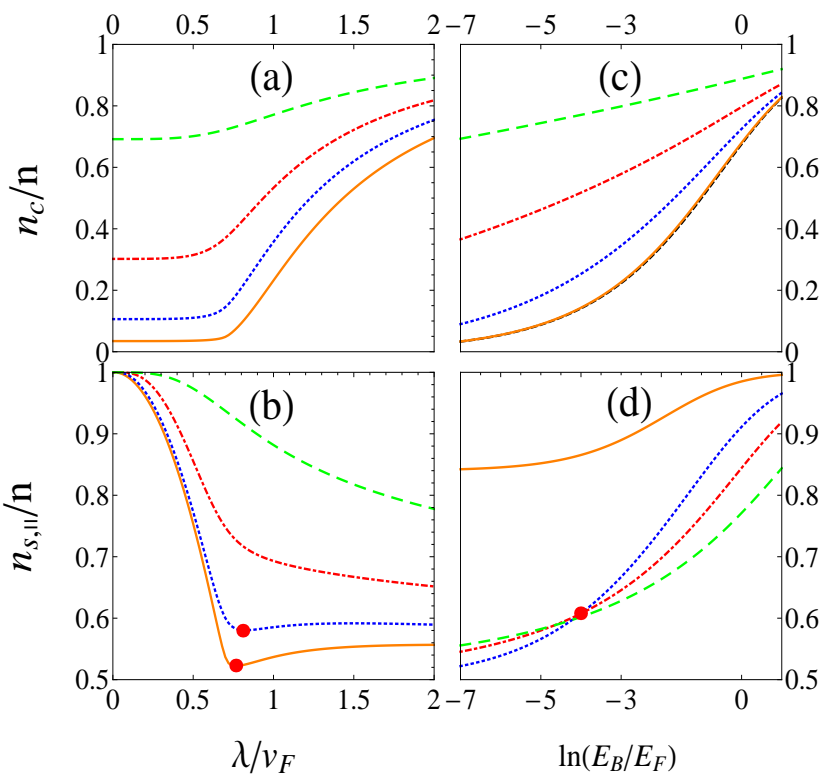

FIG. 2: (Color online) Condensed and superfluid fraction of 2D Fermi gas as functions of $\lambda / v_{F}$ and $E_{B} / E_{F}$. In (a) and (b), $E_{B} / E_{F}$ are given as: 0.001 for solid orange lines, 0.01 for dotted blue lines, 0.1 for dot-dashed red lines and 1.1 for medium-dashed green lines. In (c) and (d), $\lambda / v_{F}$ are set to be $0.4,0.8,1.2$ and 2.0 for solid orange, dotted blue, dot-dashed red, medium-dashed green lines, respectively. Dashed black line in (c) corresponds to $\lambda=0$. Red points in (b) and (d) are explained in text.

$=\ln \left(\sqrt{\tilde{\mu}^{2}+\tilde{\Delta}_{0}^{2}}-\tilde{\mu}\right)-K\left(\tilde{\mu}, \tilde{\Delta}_{0}, \tilde{\lambda}\right)$ respectively with $H\left(\tilde{\mu}, \tilde{\Delta}_{0}, \tilde{\lambda}\right)$ and $K\left(\tilde{\mu}, \tilde{\Delta}_{0}, \tilde{\lambda}\right)$ given in [20]. Results of superfluid and condensed fraction are shown in Fig.2. The same phenomena discussed in 3D are also found in 2D case. However, we find that $\lambda_{c}$ drifts leftwards when increasing $E_{B} / E_{F}$ as can be seen from Fig. 22a). The critical interaction parameter for the appearance of a minimum point of $n_{s, \|} / n$ is $E_{B} \approx 0.018 E_{F}$.

Conclusions.In summary, general formulae were obtained for the condensed density and superfluid density tensor of a two-component Fermi gases in the presence of a Rashba-type SOC. At zero temperature, we found that superfluidity in the SOC direction is suppressed while condensation is enhanced by SOC and this phenomenon becomes most pronounced for very weak interaction parameters and $\lambda>\max \left(\lambda_{c}, \lambda_{c}^{\prime}\right)$. Furthermore, the superfluid fraction exhibits a non-monotonic behavior with a minimum as a function of SOC strength when interaction parameters is below a critical value while the condensed fraction increases only monotonously with either interaction parameter or SOC strength. These phenomena happen in both $3 \mathrm{D}$ and $2 \mathrm{D}$ cases.

Finally, we point out that there is an essential difference considering the mechanism of suppressing superfluidity by disorder [21, 22] and SOC. Superfluid motion is suppressed through energy dissipation due to scattering with impurities.
While the nonzero normal density at zero temperature induced by SOC is a direct result of the triplet pairing field. In [9], it is demonstrated that, at zero temperature, this triplet pairing field induces a non-zero spin susceptibility which implies a residual normal fluid [23]. An interesting future work is the combined effect of disorder and SOC on these two phenomena where a bose-glass state [21] may show up based on the conclusion that disorder and SOC both suppress superfluidity while depletion of the condensate induced by disorder may be weakened/cancelled by SOC.

Acknowledgements. We thank Prof. L. Salasnich for helpful discussions. This work has been supported by the National Natural Science Foundation of China under Grant 50831006 and the National Basic Research Program No.2010CB934603, the Ministry of Science and Technology of China.

Note Added: After finishing this paper, we note that the full condensed density is also discussed in [24].

[1] C. L. Kane and E. J. Mele, Phys. Rev. Lett. 95, 226801 (2005); M. Z. Hasan and C. L. Kane, Rev. Mod. Phys. 82, 3045 (2010); X. L. Qi and S. C. Zhang, arXiv:1108.2026 [Rev. Mod. Phys. (to be published)].

[2] Y. A. Bychkov and E. I. Rashba, J. Phys. C 17, 6039 (1984); L. P. Gor'kov and E. I. Rashba, Phys. Rev. Lett. 87, 037004 (2001).

[3] F. S. Bergeret, A. F. Volkov, and K. B. Efetov, Rev. Mod. Phys. 77, 1321 (2005).

[4] Y.-J. Lin et al., Nature (London) 471, 83 (2011).

[5] J. D. Sau et al., Phys. Rev. B 83, 140510 (2011).

[6] J. P. Vyasanakere, S. Zhang, and V. B. Shenoy, Phys. Rev. B 84, 014512 (2011).

[7] Hui Hu et al., arXiv:1105.2488 [Phys. Rev. Lett. (to be published)].

[8] J. P. Vyasanakere and V. B. Shenoy, Phys. Rev. B 83, 094515 (2011).

[9] Li Han and C. A. R. Sa de Melo, arXiv:1106.3613

[10] M. Iskin and A. L. Subasi, Phys. Rev. Lett. 107, 050402 (2011).

[11] Z.-Q. Yu and H. Zhai, arXiv:1105.2250

[12] C. N. Yang, Rev. Mod. Phys. 34, 694 (1962); A. J. Leggett, Quantum Liquids. Bose Condensation and Cooper Pairing in Condensed-Matter Systems (Oxford University Press, Oxford, 2006).

[13] E. M. Lifshitz and L. P. Pitaevskii, Statistical Physics, Part 2 (Butterworth-Heinemann, Oxford, 2002).

[14] A. Altland and B. Simons, Condensed Matter Field Theory (Cambridge University Press, Cambridge, England, 2006).

[15] L. Dell'Anna et al., arXiv:1108.1132v1.

[16] E. Taylor, A. Griffin, N. Fukushima, and Y. Ohashi, Phys. Rev. A 74, 063626 (2006); N. Fukushima, Y. Ohashi, E. Taylor, and A. Griffin, ibid 75, 033609 (2007); Edward Taylor, Phys. Rev. B 77, 144521 (2008).

[17] M. E. Fisher, M. N. Barber, and D. Jasnow, Phys. Rev. A 8, 1111 (1973).

[18] Qizhong Zhu, Chuanwei Zhang, and Biao Wu, arXiv:1109.5811

[19] G. E. Astrakharchik, J. Boronat, J. Casulleras, and S. Giorgini, Phys. Rev. Lett. 95, 230405 (2005). 
[20] $H(\tilde{\mu}, \tilde{\Delta}, \tilde{\lambda})=\tilde{\lambda} \tilde{\Delta}^{2}[N(2 \theta, \kappa)-M(2 \theta, \kappa)] /$ $\left[4 \kappa(1-\kappa)\left(x_{0}^{2}+\tilde{\Delta}^{2}\right)^{3 / 4}\right], K(\tilde{\mu}, \tilde{\Delta}, \tilde{\lambda})=\tilde{\lambda} F(2 \theta, \kappa) /$ $\left(x_{0}^{2}+\tilde{\Delta}^{2}\right)^{1 / 4}$ where $x_{0}=\tilde{\mu}+\tilde{\lambda}^{2}, 2 \kappa=1+x_{0} / \sqrt{x_{0}^{2}+\tilde{\Delta}^{2}}$, $\tan \theta=\tilde{\lambda} /\left(x_{0}^{2}+\tilde{\Delta}^{2}\right)^{1 / 4}, N(\phi, \kappa)=E(\phi, \kappa)+(\kappa-$ 1) $F(\phi, \kappa)$, and $M(\phi, \kappa)=\kappa \sin \phi \cos \phi / \sqrt{1-\kappa \sin ^{2} \phi}$. $E(\phi, \kappa)=\int_{0}^{\phi} \sqrt{1-\kappa \sin ^{2} x} d x$ and $F(\phi, \kappa)=$ $\int_{0}^{\phi} 1 / \sqrt{1-\kappa \sin ^{2} x} d x$ are the first and second kind incomplete elliptic integrals respectively with $0 \leq \phi \leq \pi$ in this paper.

[21] K. Huang and H. F. Meng, Phys. Rev. Lett. 69, 644 (1992).

[22] G. Orso, Phys. Rev. Lett. 99, 250402(2007).

[23] A. J. Leggett, Phys. Rev. 140, A1869 (1965).

[24] L. Dell'Anna et al., arXiv:1108.1132v3; Beibing Huang, Shaolong Wan, arXiv:1109.3970 